\title{
Mathematical Reasoning of Economic Intervening Principle Based on "Yin Yang Wu Xing” Theory in Traditional Chinese Economics (I)
}

\author{
Ziqing Zhang ${ }^{1}$, Yingshan Zhang ${ }^{2}$ \\ ${ }^{1}$ No. 2 Middle School of East China Normal University, Shanghai, China \\ ${ }^{2}$ School of Finance and Statistics, East China Normal University, Shanghai, China \\ Email: ysh_zhang@163.com,yszhang@stat.ecnu.edu.cn
}

Received October 13, 2012; revised November 20, 2012; accepted December 25, 2012

\begin{abstract}
By using mathematical reasoning, this paper demonstrates the economic intervening principle: "Virtual disease is to fill his mother but real disease is to rush down his son" and "Strong inhibition of the same time, support the weak" based on "Yin Yang Wu Xing" Theory in Traditional Chinese Economics (TCE). We defined generalized relations and generalized reasoning, introduced the concept of steady multilateral systems with two non-compatibility relations, and discussed its energy properties. Later based on the intervening principle of TCE and treated the economic society as a steady multilateral system, it has been proved that the intervening principle above is true. The kernel of this paper is the existence and reasoning of the non-compatibility relations in steady multilateral systems, and it accords with the oriental thinking model.
\end{abstract}

Keywords: Traditional Chinese Economics (TCE); "Yin Yang Wu Xing” Theory; Steady Multilateral Systems; Opposite Non-Compatibility Relations; Side Effects; Imbalance of Economy; Economic Crisis; Economic Intervention Resistance Problem

\section{Main Differences between Traditional Chinese Economics and Western Economics}

In Western economics, the word "economy" comes from the Greek, its meaning "to manage a family". Materialism represents the academic on the economic law will "family" and "management" two words for the combination of economic understanding.

Western economics late nineteenth century was introduced into China, initially, "economics" to be directly translated as "rich national policy", "living", "financial management learning" words. First with the Chinese character "Jing-Ji-Xue (经济学)" translation "economics" is a Japanese, then the western Chinese translation, the word "translation" back to China by Sun Zhong-Shan (孙中山), becoming the modern Chinese of the "Jing-Ji (经济) or economy" is a word to another source.

The ancient Chinese "Jing-Ji (经济) or economy", in the 4-th century eastern time, has been officially use "economy" one word. "Jing-Ji (经济)" is a word in the first of the Zhouyi (周易), appear. The "Jing (经)" explanation "size" that refers to a broad field (vertical and horizontal fields). "Ji (济)" word from the water, explain for "crossing", that is, crossing the water.
"Jing-Ji (经济)" two words of the act, the earliest the Sui dynasty in the whole of the said "music" in the article of "economical way". Original intention is to point "managing administration serving people", and "controlling kingdom producing things", also is the world to run a meaning.

The ancients speak of "economic" in Chinese is a word to run a world. This and what we now understand the financial and economic completely different things. So, people in to the basic necessities, national wealth national politics, and other aspects of the content, it is to use what words to express? Originally used the "foods and goods" to said. "Food" refers to the agricultural production; "Goods" means agricultural sideline production and the farm currency (or money). In addition, also appeared on financial, the prosperous common people, business words. In other words, in Traditional Chinese Economics (TCE), both production and management of "foods and goods" are believed to as a complex system. It is because to run a world is difficult and complex in which there are the loving relation, the killing relation and the equivalent relation. The loving and killing relations are non-compatibility relations, which can compose the whole energy of the system greater than or less 
than the sum of each part energy of the system, rarely equal conditions. Economics means managing or controlling or intervention for the social complex system, and so on. Pursue the goal is the harmonious sustainable of the social complex system under not outward expansion of the development conditions.

But, in Western economics, economics means money and the activities related to the currency. Both production and management of money are believed to as a simple system. Money has everything. A lot of money can form a rich economic society. It is because to run a family is easy and simple in which there is only a compatibility relation or a generalized equivalent relation. The generalized equivalent relation or the compatibility relation can compose the whole energy of the system equal to the sum of each part energy of the system. Thus free and competitive can compose the family system outward expansion development. Therefore, pursue the goal is free or competition for obtaining money in order to compose the family system outward expansion development.

Western economics using free and competition treats directly economic downturn or overheating from Microscopic point of view, always destroy the original economic society's balance, and has none beneficial to economic society's immunity. Western economic intervention method can produce imbalance of economy to human society, having strong side effects. Excessively using methods of intervention economics can easily paralysis the economic society's immunity, which economic crisis is a product of Western economics. Using the method of intervention economics too little can easily produce the economic intervention resistance problem.

Traditional Chinese Economics (TCE) studies the world from the Macroscopic point of view, and its target is in order to maintain the original balance of economic society system and in order to enhance the economic society's immunity. TCE believes that each economic intervention has one-third of badness. She never encourages government to use economic intervention in long term. The ideal way is $W u W e i E r W u B u W u$ (无为而无 不为) - by doing nothing, everything is done. TCE has over 5000-year history. It has almost none side effect or economic intervention resistance problem.

After long period of practicing, our ancient economic scientists use "Yin Yang Wu Xing" Theory extensively in the traditional economic intervening principle to explain the origin of foods and goods, the law of economic society, economic changes, economic downturn or overheating diagnosis, economic crisis prevention, and so on. It has become an important part of the TCE. "Yin Yang $\mathrm{Wu}$ Xing" Theory has a strong influence to the formation and development of traditional Chinese economic theory. As is known to all, China in recent decades, economy has made great strides in development. Its reason is difficult to say the introduction of western economics, the fact that the Chinese traditional philosophy culture is in all kinds of economic decision plays a role. Her many economic intervening methods come from the traditional Chinese medicine since both human body and economic society are all complex systems. But, many Chinese and foreign scholars still have some questions on the reasoning of TCE. In this article, we will start to the western world for presentation of traditional Chinese economy introduced some mathematical and logic analysis concept.

Zhang's theories, multilateral matrix theory [1] and multilateral system theory [2-19], have given a new and strong mathematical reasoning method from macro (Global) analysis to micro (Local) analysis. He and his colleagues have made some mathematical models and methods of reasoning [20-35], which make the mathematical reasoning of TCE possible based on "Yin Yang Wu Xing" Theory [36-38]. This paper will use steady multilateral systems to demonstrate the intervening principle of TCE: "Real disease for economic over-heating is to rush down his son but virtual disease for economic downturn is to fill his mother" and "Strong inhibition of the same time, support the weak".

The article proceeds as follows. Section 2 contains basic concepts and main theorems of steady multilateral systems while the intervening principle of TCE is demonstrated in Section 3. Some discussions in TCE are given in Section 4 and conclusions are drawn in Section 5.

\section{Basic Concept of Steady Multilateral Systems}

In the real world, we are enlightened from some concepts and phenomena such as "biosphere", "food chain", "ecological balance" etc. With research and practice, by using the theory of multilateral matrices [1] and analyzing the conditions of symmetry [20-24] and orthogonality [25-35] what a stable system must satisfy, in particular, with analyzing the basic conditions what a stable working procedure of good product quality must satisfy $[9,29]$, we are inspired and find some rules and methods, then present the logic model of analyzing stability of complex systems-steady multilateral systems [2-19]. There are a number of essential reasoning methods based on the stable logic analysis model, such as "transition reasoning", "atavism reasoning", "genetic reasoning" etc. We start and still use concepts and notations in papers [3-6].

\subsection{Generalized Relations and Reasoning}

Let $V$ be a non-empty set and define its cross product as $V \times V=\{(x, y): x \in V, y \in V\}$. A generalized relation of $V$ is a non-empty subset $R \subset V \times V$. TCE mainly researches generalized relation rules for general $V$ rather than for special $V$. 
For a relation set $\mathfrak{R}=\left\{R_{0}, \cdots, R_{m-1}\right\}$, define both an inverse relationship of $R_{\mathrm{i}} \in \mathfrak{R}$ and a relation multiplication between $R_{\mathrm{i}} \in \mathfrak{R}$ and $R_{j} \in \mathfrak{R}$ as follows:

$$
R_{i}^{-1}=\left\{(x, y):(y, x) \in R_{i}\right\}
$$

And

$R_{i} * R_{j}=\{(x, y):$ there is at least an $u \in V$ such that $(x, u) \in R_{i}$ and $\left.(u, y) \in R_{j}\right\}$.

The relation $R_{\mathrm{i}} \in \mathfrak{R}$ is called reasonable if $R_{i}^{-1} \in \mathfrak{R}$. A generalized reasoning of general $V$ is defined as for $R_{i} * R_{j} \neq \varnothing$ there is a relation $R_{k} \in \mathfrak{R}$ such that $R_{i} * R_{j} \subset R_{k}$.

The generalized reasoning satisfies the associative law of reasoning, i.e., $\left(R_{i} * R_{j}\right) * R_{k}=R_{i} *\left(R_{j} * R_{k}\right)$. This is the basic requirement of reasoning in TCE. But there are a lot of reasoning forms which do not satisfy the associative law of reasoning in Western Science. For example, in true and false binary of proposition logic, the associative law does not hold on its reasoning because

$$
\begin{aligned}
& (\text { false } * \text { false }) * \text { false }=\text { true } * \text { false }=\text { false } \\
& \neq \text { true }^{*} \text { false } e^{*} \text { true }=\text { false }{ }^{*}\left(\text { false } \text { false }^{*}\right.
\end{aligned}
$$

\subsection{Equivalence Relations}

Let $V$ be a non empty set and $R_{0}$ be its a relation. We call it an equivalence relation, denoted by $\sim$, if the following three conditions are all true:

1) Reflexive: $(x, x) \in R_{0}$ for all $x \in V$, i.e., $x \sim x$;

2) Symmetric: if $(x, y) \in R_{0}$, then $(y, x) \in R_{0}$, i.e., if $x \sim y$, then $y \sim x$;

3) Conveyable (Transitivity): if $(x, y) \in R_{0},(y, z) \in R_{0}$, then $(x, y) \in R_{0}$, i.e., if $x \sim y, y \sim z$, then $x \sim z$.

Furthermore, the relation $R$ is called a compatibility relation if there is a non-empty subset $R_{1} \subset R$ such that $R_{1}$ satisfies at least one of the conditions above. And the relation $R$ is called a non-compatibility relation if there doesn't exist any non-empty subset $R_{1} \subset R$ such that $R_{1}$ satisfies any one of the conditions above. Any one of compatibility relations can be expanded into an equivalent relation to some extent [2].

Western Science only considers the reasoning under one Axiom system such that only compatibility relation reasoning is researched. However there are many Axiom systems in Nature. Traditional Chinese Science mainly researches the reasoning among many Axiom systems in Nature. Of course, she also considers the reasoning under one Axiom system but she only expands the reasoning as the equivalence relation reasoning.

\subsection{Two Kinds of Opposite Non-Compatibility Relations}

Equivalent relations, even compatibility relations, cannot portray the structure of the complex systems clearly. For example, assume that $A$ and $B$ are good friends and they have close relations. So are $B$ and $C$. However, you cannot get the conclusion that $A$ and $C$ are good friends.

We denote $A \rightarrow B$ as that $A$ and $B$ have close relations. Then the example above can be denoted as: $A \rightarrow B, B \rightarrow C$ do not imply $A \rightarrow C$, i.e., the relation $\rightarrow$ is a non-conveyable (or non-transitivity) relation, of course, a non-equivalent relation. In the following, we consider two non-compatibility relations.

In TCE, any Axiom system is not considered, but should first consider using a logic system. Believe that the rules of Heaven and the behavior of Human can follow the same logic system (天人合一). This logic system is equivalent to a group of computation. The method is to take the research object classification following the selected logic system, without considering the specific content of the research object, namely classification taking images (比类取象). Analysis of the relationship between research objects, make relationships with computational reasoning comply with the selected logic system operation, and then in considering the research object of the specific content of the conditions, according to the logic of the selected system operation to solve specific problems. In mathematics, the method of classification taking images is explained in the following Definition 2.1.

Definition 2.1. Suppose that there exists a finite group $G^{m}=\left\{g_{0}, \cdots, g_{m-1}\right\}$ of order $m$ where $g_{0}$ is identity. Let $V$ be a none empty set satisfying that $V=V_{g_{0}}+\cdots+V_{g_{m-1}}$ where the notation means that $V=V_{g_{0}}^{g_{0}} \cup \cdots \cup V_{g_{m-1}}, V_{g_{i}} \cap V_{g_{j}}=\varnothing, \forall i \neq j$ (the following the same). The $V_{g_{j}}$ is called a factor image or data image of group element $g_{j}$ for any $j$ if $V_{g_{j}}$ is factor or data.

Denoted $V_{g_{i}} \times V_{g_{j}}=\left\{(x, y): x \in V_{g_{i}}, y \in V_{g_{j}}\right\}$, where the note $x$ is the usual Cartesian product or cross join. Define relations

$$
R_{g_{r}}=\sum_{g \in G^{m}} V_{g} \times V_{g g_{r}}, r=0, \cdots, m-1,
$$

where $R_{g_{0}}=R_{g_{0}}^{-1}=R_{g_{0}^{-1}}$ is called an equivalence relation of $V$ if $g_{0}$ is identity; denoted by ; $R_{g_{s}}=R_{g_{s}}^{-1}=R_{g_{s}^{-1}}$ is called a symmetrical relation of $V$ if $g_{s}=g_{s}^{-1}, s \neq 0$; denoted by $\stackrel{R_{s}}{\longleftrightarrow}$ or $\leftrightarrow$; $R_{g_{1}}=R_{g_{1}^{-1}}^{-1}$ is called a neighboring relation of $V$ if $g_{1} \neq g_{1}^{-1} ;$ denoted by $\stackrel{R_{1}}{\longrightarrow}$ or $\rightarrow$;

$R_{g_{a}}=R_{g_{a}^{-1}}^{-1} \neq R_{g_{a}^{-1}}, R_{g_{1}}, R_{g_{1}^{-1}}$ is called an alternate (or 
atavism) relation of $V$ if $g_{a} \neq g_{a}^{-1}, g_{1}, g_{1}^{-1}, a \geq 2$; denoted by $\stackrel{R_{a}}{\Rightarrow}$ or $\Rightarrow$.\#

In this case, the equivalence relations and symmetrical relations are compatibility relations but both neighboring relations and alternate relations are non-compatibility relations. For the given relation set $\mathfrak{R}=\left\{R_{g_{0}}, \cdots, R_{g_{m-1}}\right\}$, these relations are all reasoning relations since the relation $R_{g_{i}}^{-1}=R_{g^{-1}} \in \mathfrak{R}$ if $R_{g_{i}} \in \mathfrak{R}$.

The equivălence relation $R_{g_{0}}$, symmetrical relations $R_{g_{s}}$, neighboring relation $R_{g_{1}}$ and alternate relations $R_{g_{a}}$ are all the possible relations for the method of classification taking images. In this paper, we mainly consider the equivalence relation $R_{g_{0}}$, neighboring relation $R_{g_{1}}$ and alternate relations $R_{g_{a}}$.

There is an unique generalized reasoning between the two kinds of opposite non-compatibility relations for case $m=5$. For example, let $V$ be a none empty set, there are two kinds of opposite relations: the neighboring relation $R_{1}$, denoted $(x, y) \in R_{1}$ by $x \rightarrow y$ and the alternate (or atavism) relation $R_{2}$, denoted $(x, y) \in R_{2}$ by $x \Rightarrow y$, the logic reasoning architecture [2-19] of "Yin Yang Wu Xing" Theory in Ancient China is equivalent to the following reasoning:

1) If $(x, y) \in R_{1}, \quad(y, z) \in R_{1}$, then $(x, y) \in R_{2}$;

$\Leftrightarrow$ if $(x, y) \in R_{1}, \quad(y, z) \in R_{2}$, then $(y, z) \in R_{1}$;

$\Leftrightarrow$ if $(x, z) \in R_{2},(y, z) \in R_{1}$ then $(x, y) \in R_{1}$;

2) If $(x, y) \in R_{2},(y, z) \in R_{2}$, then $(z, x) \in R_{1}$;

$\Leftrightarrow$ if $(z, x) \in R_{1}, \quad(x, y) \in R_{2}$, then $(y, z) \in R_{2}$;

$\Leftrightarrow$ if $(y, z) \in R_{2},(z, x) \in R_{1}$, then $(x, y) \in R_{2}$.

The reasoning is also equivalent to the following calculating

$$
R_{i} * R_{j} \subset R_{\bmod (i+j, 5)}, \forall i, j \in\{1,2,3,4\},
$$

where $R_{4}=R_{1}^{-1}, R_{3}=R_{2}^{-1}$ and the $\bmod (i+j, 5)$ is the addition of module 5 .

The two kinds of opposite relations can not be exist separately. Such reasoning can be expressed in Figure 1. The first triangle reasoning is known as a jumpingtransition reasoning, while the second triangle reasoning is known as an atavism reasoning. Reasoning method is a triangle on both sides decided to any third side. Both neighboring relations and alternate relations are not compatibility relations, of course, none equivalence relations, called non-compatibility relations.

\subsection{Genetic Reasoning}

Let $V$ be a none empty set with the equivalent relation $R_{0}$, the neighboring relation $R_{1}$ and the alternate relations $R_{a} \neq R_{a}^{-1}, R_{1}^{-1}, R_{1}, a \geq 2$ denoted by $\sim, \rightarrow$ and $\Rightarrow$, respectively. Then a genetic reasoning is defined as follows:
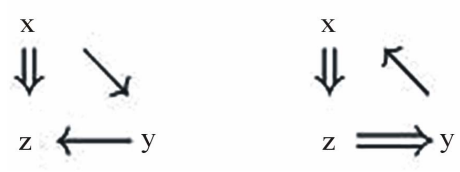

Figure 1. Triangle reasoning.

1) if $x \sim y, \quad y \rightarrow z$, then $x \rightarrow z$;

2) if $x \sim y, y \Rightarrow z$, then $x \Rightarrow z$;

3) if $x \rightarrow y, y \sim z$, then $x \rightarrow z$;

4) if $x \Rightarrow y, y \sim z$, then $x \Rightarrow z$.

The reasoning is also equivalent to the following calculating

$$
R_{0} * R_{j}=R_{j} * R_{0}=R_{j}, \forall j \in\{0,1, \cdots, m-1\}=G_{0}^{m}
$$

where $G_{0}^{m}=\{0, \cdots, m-1\}$ is a finite group of order $m$. The genetic reasoning is equivalent to that there is a group $G_{0}^{m}=\{0, \cdots, m-1\}$ with the operation * such that $V$ can be cut into $V=V_{0}+\cdots+V_{m-1}$ where $V_{i}$ may be an empty set and the corresponding relations of reasoning can be written as the forms as follows

$$
R_{r}=\sum_{i=0}^{m-1} V_{i} \times V_{i^{*} r}, r=0, \cdots, m-1,
$$

Satisfying $R_{i}^{*} R_{j} \subset R_{i^{*} j}, \forall i, j \in G_{0}^{m}$.

\subsection{Steady Multilateral Systems}

For a none empty set $V$ and its a relation set $\mathfrak{R}=\left\{R_{0}, \cdots, R_{m-1}\right\}$, the form $(V, \mathfrak{R})$ (or simply, $\left.V\right)$ is called a multilateral system [2-19], if $(V, \mathfrak{R})$ satisfies the following properties:

1) $R_{0}+\cdots+R_{m-1} \subset V \times V$, i.e. $R_{i} \cap R_{j}=\varnothing, \forall i \neq j$.

2) $R_{0} * R_{j}=R_{j} * R_{0}=R_{j}, \forall j \in\{0,1, \cdots, m-1\}=G_{0}^{m}$

3) The relation $R_{i}^{-1} \in \mathfrak{R}$ if $R_{i} \in \Re$.

4) For $R_{i} * R_{j} \neq \varnothing$, there is a relation $R_{k} \in \Re$ such that $R_{i} * R_{j} \subset R_{k}$.

The 4) is called the reasoning, the 1) the uniqueness of reasoning, the 2) the hereditary of reasoning (or genetic reasoning) and the 3 ) the equivalent property of reasoning of both relations $R_{i} \in \Re$ and $R_{i}^{-1} \in \Re$, i.e., the reasoning of $R_{i} \in \mathfrak{R}$ is equivalent to the reasoning of $R_{i}^{-1} \in \Re$. In this case, the two-relation set $\left\{R_{i}, R_{i}^{-1}\right\}$ is a lateral relation of $(V, \Re)$. The $R_{0}$ is called an equivalence relation. The multilateral system $(V, \mathfrak{R})$ can be written as $\left(V_{0}+\cdots+V_{n-1},\left\{R_{0}, \cdots, R_{m-1}\right\}\right)$. Furthermore, the $V$ and $\mathfrak{R}$ are called the state space and relation set considered of $(V, \Re)$, respectively. For a multilateral system $(V, \Re)$, it is called complete (or, perfect) if " $\subset$ " changes into " $="$. And it is called complex if there exists at least a non-compatibility relation $R_{i} \in \mathfrak{R}$. In this case, the multilateral system is also called a logic analysis model of complex systems.

Let $R_{1}$ be a non-compatibility relation. A complex multilateral system 
$(V, \mathfrak{R})=\left(V_{0}+\cdots+V_{n-1},\left\{R_{0}, \cdots, R_{m-1}\right\}\right)$ is said as a steady multilateral system (or, a stable multilateral system) if there exists a number $n$ such that $R_{1}^{* n}=R_{0}$ where $R_{1}^{* n}=R_{1}^{* * *} R_{1}$. The condition is equivalent to there is a the chain $x_{1}, \cdots, x_{n} \in V$ such that $\left(x_{1}, x_{2}\right) \in R_{1}, \cdots,\left(x_{n}, x_{1}\right) \in R_{1}$, or $x_{1} \rightarrow x_{2} \rightarrow \cdots \rightarrow x_{n} \rightarrow x_{1}$. The steady multilateral system is equivalent to the complete multilateral system. The stability definition given above, for a relatively stable system, is most essential. If there is not the chain or circle, then there will be some elements without causes or some elements without results in a system. Thus, this system is to be in the state of finding its results or causes, i.e., this system will fall into an unstable state, and there is not any stability to say.

Theorem 2.1. The system $(V, \Re)$ is a multilateral system if and only if there exists a finite group $G^{m}=\left\{g_{0}, \cdots, g_{m-1}\right\}$ of order $m$ where $g_{0}$ is identity such that the relation set $\mathfrak{R}=\left\{R_{g_{0}}, \cdots, R_{g_{m-1}}\right\}$ satisfying $R_{g_{i}} * R_{g_{j}} \subset R_{g_{i} g_{j}}, \forall i, j \in\{0,1, \cdots, m-1\} . \#$

In this case, the multilateral system $(V, \Re)$ can be written as $\left(V_{g_{0}}+\cdots+V_{g_{m-1}},\left\{R_{g_{0}}, \cdots, R_{g_{m-1}}\right\}\right)$ satisfying $R_{g_{r}}=\sum_{g \in G^{m}} V_{g} \times V_{g g_{r}}, r=0, \cdots, m-1$, where $V_{g_{i}}$ may be an empty set.

Theorem 2.2. If the multilateral system $(V, \Re)=\left(V_{0}+\cdots+V_{n-1},\left\{R_{0}, \cdots, R_{m-1}\right\}\right)$ is a steady multilateral system, then $n=m$ and $\mathfrak{R}=\left\{R_{0}, \cdots, R_{m-1}\right\}$ is a finite group of order $m$ about the relation multiplication $R_{i} * R_{j}=R_{k} \quad$ where $V_{i} \quad$ must be a non empty set.\#

Definition 2.2. Suppose that a multilateral system $(V, \Re)$ can be written as

$$
\begin{aligned}
\left(V_{g_{0}}+\cdots+V_{g_{m-1}},\left\{R_{g_{0}}, \cdots, R_{g_{m-1}}\right\}\right) & \text { satisfying } \\
& R_{g_{r}}=\sum_{g \in G^{m}} V_{g} \times V_{g g_{r}}, r=0, \cdots, m-1
\end{aligned}
$$

and $R_{g_{i}} * R_{g_{j}} \subset R_{g_{i} g_{j}}, \forall i, j \in\{0,1, \cdots, m-1\}$.

The group $G^{m}=\left\{g_{0}, \cdots, g_{m-1}\right\}$ of order $m$ where $g_{0}$ is identity is called the representation group of the multilateral system $(V, \mathfrak{R})$. The representing function of $R_{g_{r}}$ is defined as follows

$$
I\left(R_{g_{r}}\right)=\left\{(x, y): x^{-1} y=g_{r}, x, y \in G^{m}\right\}, r=0, \cdots, m-1
$$

Let multilateral systems $\left(V^{i}, \mathfrak{R}^{i}\right), i=1,2$ be with two representation groups $G_{i}, i=1,2$, respectively. Both multilateral systems $\left(V^{i}, \mathfrak{R}^{i}\right), i=1,2$ are called isomorphic if the two representation groups $G_{i}, i=1,2$ are isomorphic.\#

Theorems 2.1 and 2.2 and Definitions 2.1 and 2.2 are key concepts in multilateral system theory because they show the classification taking images as the basic method. In the following, introduce two basic models to illustrate the method.

Theorem 2.3. Suppose that $G_{0}^{2}=\{0,1\}$ with multiplication table

\begin{tabular}{lll}
\hline$*$ & 0 & 1 \\
\hline 0 & 0 & 1 \\
1 & 1 & 0 \\
\hline
\end{tabular}

i.e., the multiplication of $G_{0}^{2}$ is the addition of module 2. In other words, $i^{*} j=\bmod (i+j, 2)$.

And assume that

$$
(V, \Re)=\left(V_{0}+V_{1},\left\{R_{0}, R_{1}\right\}\right)
$$

satisfying

$$
\begin{gathered}
R_{r}=\sum_{i=0}^{1} V_{i} \times V_{\bmod (i+r, 2)}, r=0,1, \\
R_{i} * R_{j} \subset R_{\bmod (i+j, 2)}, \forall i, j \in G_{0}^{2} .
\end{gathered}
$$

Then $(V, \Re)$ is a steady multilateral system with one equivalent relation $R_{0}$ and one symmetrical relation $R_{1}$ which is a simple system since there is not any non-compatibility relation. In other words, the relations $R_{i}$ 's are the simple forms as follows:

$$
I\left(R_{0}\right)=\{(0,0),(1,1)\}, I\left(R_{1}\right)=\{(0,1),(1,0)\}
$$

where $(i, j)$ is corresponding to $V_{i} \times V_{j}$.\#

It will be proved that the steady multilateral system in Theorem 2.3 is the reasoning model of "Tao" (道) in TCE if there are two energy functions $\varphi\left(V_{0}\right)$ and $\varphi\left(V_{1}\right)$ satisfying $\varphi\left(V_{0}\right)>\varphi\left(V_{1}\right)$, called Dao model, denoted by $V^{2}$.

Theorem 2.4. For each element $x$ in a steady multilateral system $V$ with two non-compatibility relations, there exist five equivalence classes below:

$$
\begin{aligned}
& X=\{y \in V \mid y \sim x\}, X_{S}=\{y \in V \mid x \rightarrow y\}, \\
& X_{S}=\{y \in V \mid x \rightarrow y\}, X_{K}=\{y \in V \mid x \Rightarrow y\}, \\
& K_{X}=\{y \in V \mid y \Rightarrow x\}, S_{X}=\{y \in V \mid y \rightarrow x\}
\end{aligned}
$$

Which the five equivalence classes have relations in Figure 2.\#

It can be proved that the steady multilateral system in Theorem 2.4 is the reasoning model of "Yin Yang Wu Xing" in TCE if there are five energy functions (Defined in Section 3) $\varphi(X), \varphi\left(X_{S}\right), \varphi\left(X_{K}\right), \varphi\left(K_{X}\right)$ and $\varphi\left(S_{X}\right)$ satisfying

$$
\varphi\left(X_{K}\right)>\varphi\left(X_{S}\right)>\varphi(X)>\varphi\left(X_{K}\right)>\varphi\left(S_{X}\right)
$$

Called Wu-Xing model, denoted by $V^{5}$.

By Definition 2.2, the Wu-Xing model $V^{5}$ can be 


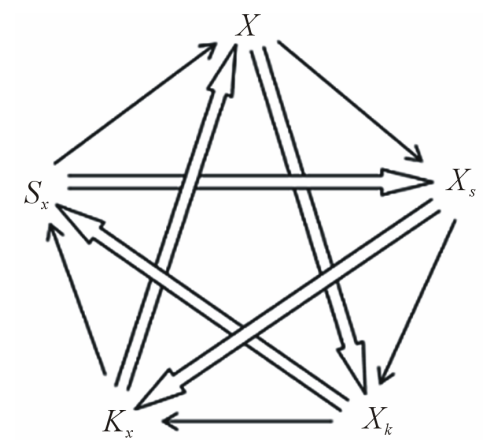

Figure 2. The method of finding Wu-Xing.

written as follows:

Define $\quad V_{0}=X, \quad V_{1}=X_{S}, \quad V_{2}=X_{K}, \quad V_{3}=K_{X}$, $V_{4}=S_{X}$, corresponding to wood, fire, soil, metal, water, and assume

$$
V=V_{0}+V_{1}+\cdots+V_{4}
$$

and

$$
\Re=\left\{R_{0}, R_{1}, \cdots, R_{4}\right\}
$$

satisfying

$$
\begin{gathered}
R_{r}=\sum_{i=0}^{4} V_{i} \times V_{\bmod (i+r, 5)}, r \in G_{0}^{5} \\
R_{i} * R_{j} \subset R_{\bmod (i+j, 5)}, \forall i, j \in G_{0}^{5}
\end{gathered}
$$

i.e., the relation multiplication of $V^{5}$ is isomorphic to the addition of module 5. Then $V^{5}$ is a steady multilateral system with one equivalent relation $R_{0}$ and two non-compatibility relations $R_{1}=R_{4}^{-1}$ and $R_{2}=R_{3}^{-1}$.

These Theorems can been found in [1-6,11-16]. Figure in Theorem 2.4 is the Figure of "Yin Yang Wu Xing" Theory in Ancient China. The steady multilateral system $V$ with two non-compatibility relations is equivalent to the logic architecture of reasoning model of "Yin Yang Wu Xing" Theory in Ancient China. What describes the general method of complex systems can be used in economic complex system.

\section{Energy Relationship Analysis of Steady Multilateral Systems}

\subsection{Energy of a Multilateral System}

Energy concept is an important concept in Physics. Now, we introduce this concept to the multilateral systems (or economic society) and use these concepts to deal with the multilateral system diseases (economic downturn or economic overheating). In mathematics, a multilateral system is said to have Energy (or Dynamic) if there is a none negative function $\varphi(*)$ which makes every subsystem meaningful of the multilateral system.

For two subsystems $V_{i}$ and $V_{j}$ of multilateral system $V$, denote $V_{i} \rightarrow V_{j}$ (or $V_{i} \Rightarrow V_{j}$, or $V_{i} \sim V_{j}$, or
$V_{i} \leftrightarrow V_{j}$ ) means $x_{i} \rightarrow x_{j}, \forall x_{i} \in V_{i}, x_{j} \in V_{j}$ (or

$x_{i} \Rightarrow x_{j}, \forall x_{i} \in V_{i}, x_{j} \in V_{j}$, or $x_{i} \sim x_{j}, \forall x_{i} \in V_{i}, x_{j} \in V_{j}$,

or $\left.x_{i} \leftrightarrow x_{j}, \forall x_{i} \in V_{i}, x_{j} \in V_{j}\right)$.

For subsystems $V_{i}$ and $V_{j}$ where

$V_{i} \cap V_{j}=\varnothing, \forall i \neq j$. Let $\varphi\left(V_{i}\right), \varphi\left(V_{j}\right)$ and

$\varphi\left(V_{i}, V_{j}\right)$ be the energy function of $V_{i}$, the energy function of $V_{j}$ and the total energy of both $V_{i}$ and $V_{j}$, respectively.

For an equivalence relation $V_{i} \sim V_{j}$, if $\varphi\left(V_{i}, V_{j}\right)=\varphi\left(V_{i}\right)+\varphi\left(V_{j}\right)$ (the normal state of the energy of $\left.V_{i} \sim V_{j}\right)$, then the neighboring relation $V_{i} \sim V_{j}$ is called that $V_{i}$ likes $V_{j}$ which means that $V_{i}$ is similar to $V_{j}$. In this case, the $V_{i}$ is also called the brother of $V_{j}$ while the $V_{j}$ is also called the brother of $V_{i}$. In the causal model, the $V_{i}$ is called the similar family member of $V_{j}$ while the $V_{j}$ is also called the similar family member of $V_{i}$. There are not any causal relation considered between $V_{i}$ and $V_{j}$.

For a symmetrical relation $V_{i} \leftrightarrow V_{j}$, if

$$
\varphi\left(V_{i}, V_{j}\right)=\varphi\left(V_{j}, V_{i}\right)=\varphi\left(V_{i}\right)+\varphi\left(V_{j}\right)+\tau\left(V_{i}, V_{j}\right)
$$

(The normal state of the energy of $V_{i} \leftrightarrow V_{j}$ ) where the $\tau\left(V_{i}, V_{j}\right)$ is an interaction of $V_{i}$ and $V_{j}$ satisfiing $\tau\left(V_{i}, V_{j}\right)=\tau\left(V_{j}, V_{i}\right)$, then the symmetrical relation $V_{i} \leftrightarrow V_{j}$ is called that $V_{i}$ is corresponding to $V_{j}$ which means that $V_{i}$ is positively (or non-negatively) corresponding to $V_{j}$ if $\tau\left(V_{i}, V_{j}\right)>0$ (or $\tau\left(V_{i}, V_{j}\right) \geq 0$ ) and that $V_{i}$ is negatively corresponding to $V_{j}$ if $\tau\left(V_{i}, V_{j}\right)<0$. In this case, the $V_{i}$ is also called the counterpart of $V_{j}$ while the $V_{j}$ is also called the counterpart of $V_{i}$. In the causal model, the $V_{i}$ is called the reciprocal causation of $V_{j}$ while the $V_{j}$ is also called the reciprocal causation of $V_{i}$. There is a reciprocal causation relation considered between $V_{i}$ and $V_{j}$.

For an neighboring relation $V_{i} \rightarrow V_{j}$, if $\varphi\left(V_{i}, V_{j}\right)>\varphi\left(V_{j}, V_{i}\right)>\varphi\left(V_{i}\right)+\varphi\left(V_{j}\right)$ (the normal state of the energy of $\left.V_{i} \rightarrow V_{j}\right)$, then the neighboring relation $V_{i} \rightarrow V_{j}$ is called that $V_{i}$ bears (or loves) $V_{j}$ [or that $V_{j}$ is born by (or is loved by) $V_{j}$ ] which means that $V_{i}$ is beneficial on $V_{j}$ each other. In this case, the $V_{i}$ is called the mother of $V_{j}$ while the $V_{j}$ is called the son of $V_{i}$. In the causal model, the $V_{i}$ is called the beneficial cause of $V_{j}$ while the $V_{j}$ is called the beneficial effect of $V_{i}$.

For an alternate relation $V_{i} \Rightarrow V_{j}$, if $\varphi\left(V_{i}, V_{j}\right)<\varphi\left(V_{j}, V_{i}\right)<\varphi\left(V_{i}\right)+\varphi\left(V_{j}\right)$ (the normal state of the energy of $V_{i} \Rightarrow V_{j}$ ), then the alternate relation $V_{i} \Rightarrow V_{j}$ is called as that $V_{i}$ kills (or hates) $V_{j}$ [or that $V_{j}$ is killed by (or is hated by) $V_{i}$ ] which means that $V_{i}$ is harmful on $V_{j}$ each other. In this case, the $V_{i}$ is called the bane of $V_{j}$ while the $V_{j}$ is called 
the prisoner of $V_{i}$. In the causal model, the $V_{i}$ is called the harmful cause of $V_{j}$ while the $V_{j}$ is called the harmful effect of $V_{i}$.

In the future, if not otherwise stated, any equivalence relation is the liking relation, any symmetrical relation is the reciprocal causation relation, any neighboring relation is the born relation (or the loving relation), and any alternate relation is the killing relation.

Suppose $V$ is a steady multilateral system having energy, then $V$ in the multilateral system during normal operation, its energy function for any subsystem of the multilateral system has an average (or expected value in Statistics), this state is called normal when the energy function is nearly to the average. Normal state is the better state.

That a subsystem of a multilateral system is not running properly (or economic downturn, economic overheating, abnormal), is that the energy deviation from the average of the subsystems is too large (or too big), the high (real disease for economic overheating) or the low (virtual disease for economic downturn). Both economic overheating and economic downturn are all diseases of economic society.

In a subsystem of a multilateral system being not running properly, if this sub-system through the energy of external forces increase or decrease, making them return to the average (or expected value), this method is called intervention (or making an economic controlling) to the multilateral system.

The purpose of intervention is to make the multilateral system return to normal state. The method of intervention is to increase or decrease the energy of a subsystem.

What kind of intervening should follow the principle to treat it? Western economics emphasizes direct intervening, but the indirect intervening of oriental economics is required. In mathematics, which is more reasonable?

Based on this idea, many issues are worth further discussion. For example, if an economic intervening has been done to an economic society, what situation will happen?

\subsection{Intervention Rule of a Multilateral System}

For a steady multilateral system $V$ with two non-compatibility relations, suppose that there is an external force (or an intervening force) on the subsystem $X$ of $V$ which makes the energy $\varphi(X)$ of $X$ changed by the increment $\Delta \varphi(X)$, then the energies $\varphi\left(X_{S}\right), \varphi\left(X_{K}\right)$, $\varphi\left(K_{X}\right), \varphi\left(S_{X}\right)$ of other subsystems $X_{S}, X_{K}, K_{X}$, $S_{X}$ (defined in Theorem 2.4) of $V$ will be changed by the increments $\Delta \varphi\left(X_{S}\right), \Delta \varphi\left(X_{K}\right), \Delta \varphi\left(K_{X}\right)$ and $\Delta \varphi\left(S_{X}\right)$, respectively.

It is said that the multilateral system has the capability of intervention reaction if the multilateral system has capability to response the intervention force.
If a subsystem $X$ of multilateral system $V$ is intervened, then the energies of the subsystems $X_{S}$ and $S_{X}$ which have neighboring relations to $X$ will change in the same direction of the force outside on $X$. We call them beneficiaries. But the energies of the subsystems $X_{K}$ and $K_{X}$ which have alternate relations to $X$ will change in the opposite direction of the force outside on $X$. We call them victims.

In general, there is an essential principle of intervention: any beneficial subsystem of $X$ changes in the same direction of $X$, and any harmful subsystem of $X$ changes in the opposite direction of $X$. The size of the energy changed is equal, but the direction opposite.

Intervention Rule: In the case of virtual disease for economic downturn, the intervening method of intervention is to increase the energy. If the intervening has been done on $X$, the energy increment (or, increase degree) $\left|\Delta \varphi\left(X_{S}\right)\right|$ of the son $X_{S}$ of $X$ is greater than the energy increment $\left|\Delta \varphi\left(S_{X}\right)\right|$ of the mother $S_{X}$ of $X$, i.e., the best beneficiary is the son $X_{S}$ of $X$. But the energy decrease degree $\left|\Delta \varphi\left(X_{K}\right)\right|$ of the prisoner $X_{K}$ of $X$ is greater than the energy decrease degree $\left|\Delta \varphi\left(K_{X}\right)\right|$ of the bane of $X$, i.e., the worst victim is the prisoner $X_{K}$ of $X$.

In the case of real disease for economic overheating, the intervening method of intervention is to decrease the energy. If the intervening has been done on $X$, the energy decrease degree $\left|\Delta \varphi\left(S_{X}\right)\right|$ of the mother $S_{X}$ of $X$ is greater than the energy decrease degree $\left|\Delta \varphi\left(X_{S}\right)\right|$ of the son of $X$, i.e., the best beneficiary is the mother $S_{X}$ of $X$. But the energy increment $\left|\Delta \varphi\left(K_{X}\right)\right|$ of the bane $K_{X}$ of $X$ is greater than the energy increment $\left|\Delta \varphi\left(X_{K}\right)\right|$ of the prisoner $X_{K}$ of $X$, i.e., the worst victim is the bane $K_{X}$ of $X$.

In mathematics, the changing laws are as follows.

1) If $\Delta \varphi(X)=\Delta>0$, then $\Delta \varphi\left(X_{S}\right)=\rho_{1} \Delta$, $\Delta \varphi\left(X_{K}\right)=-\rho_{1} \Delta, \Delta \varphi\left(K_{X}\right)=-\rho_{2} \Delta, \Delta \varphi\left(S_{X}\right)=\rho_{2} \Delta$;

2) If $\Delta \varphi(X)=-\Delta<0$, then $\Delta \varphi\left(X_{S}\right)=-\rho_{2} \Delta$, $\Delta \varphi\left(X_{K}\right)=\rho_{2} \Delta, \Delta \varphi\left(K_{X}\right)=\rho_{1} \Delta, \Delta \varphi\left(S_{X}\right)=-\rho_{1} \Delta ;$ where $1 \geq \rho_{1} \geq \rho_{2} \geq 0$. Both $\rho_{1}$ and $\rho_{2}$ are called intervention reaction coefficients, which are used to represent the capability of intervention reaction. The larger $\rho_{1}$ and $\rho_{2}$, the better the capability of intervention reaction. The state $\rho_{1}=\rho_{2}=1$ is the best state but the state $\rho_{1}=\rho_{2}=0$ is the worst state.

This intervention rule is similar to force and reaction in Physics. In other words, if a subsystem of multilateral system $V$ has been intervened, then the energy of subsystem which has neighboring relation changes in the same direction of the force, and the energy of subsystem which has alternate relation changes in the opposite direction of the force. The size of the energy changed is equal, but the direction opposite.

In general, $\rho_{1}$ and $\rho_{2}$ are decreasing functions of 
the intervention force $\Delta$ since the intervention force $\Delta \varphi(X)$ is easily to transfer all if $\Delta$ is small but the intervention force is not easily to transfer all if $\Delta$ is large. The energy functions of complex system, the stronger the more you use. In order to magnify $\rho_{1}$ and $\rho_{2}$, should set up an economic society of the intervenetion reaction system, and often use it.

Economic intervening resistance problem is that such a question, beginning more appropriate economic intervening method, but is no longer valid after a period. It is because the capability of intervention reaction is bad, i.e., the intervention reaction coefficients $\rho_{1}$ and $\rho_{2}$ are too small. In the state $\rho_{1}=\rho_{2}=1$, any economic intervening resistance problem is non-existence but in the state $\rho_{1}=\rho_{2}=0$, economic intervening resistance problem is always existence. At this point, the paper advocates the essential principle of intervening to avoid economic intervening resistance problems.

\subsection{Self-Protection Rule of a Multilateral System}

If there is an intervening force on the subsystem $X$ of a steady multilateral system $V$ which makes the energy $\varphi(X)$ changed by increment $\Delta \varphi(X)$ such that the energies $\varphi\left(X_{S}\right), \varphi\left(X_{K}\right), \varphi\left(K_{X}\right), \varphi\left(S_{X}\right)$ of other subsystems $X_{S}, X_{K}, K_{X}, S_{X}$ (defined in Theorem 2.4) of $V$ will be changed by the increments $\Delta \varphi\left(X_{S}\right)$, $\Delta \varphi\left(X_{K}\right), \Delta \varphi\left(K_{X}\right), \Delta \varphi\left(S_{X}\right)$, respectively, then can the multilateral system $V$ has capability to protect the worst victim to restore?

It is said that the steady multilateral system has the capability of self-protection if the multilateral system has capability to protect the worst victim to restore. The capability of self-protection of the steady multilateral system is said to be better if the multilateral system has capability to protect all the victims to restore.

In general, there is an essential principle of self-protection: any harmful subsystem of $X$ should be protected by using the same intervention force but any beneficial subsystem of $X$ should not.

Self-protection Rule: In the case of virtual disease for economic downturn, the intervening method of intervention is to increase the energy. If the intervening has been done on $X$ by the increment $\Delta \varphi(X)=\Delta>0$, the worst victim is the prisoner $X_{K}$ of $X$ which has the increment $\left(-\rho_{1} \Delta\right)$. Thus the intervening principle of self-protection is to restore the prisoner $X_{K}$ of $X$ and the restoring method of self-protection is to increase the energy $\varphi\left(X_{K}\right)$ of the prisoner $X_{K}$ of $X$ by using the intervention force on $X$ according to the intervention rule. In general, the increase degree is $\left(\rho_{3} \Delta\right)$ where $\rho_{3} \leq \rho_{1}$.

In the case of real disease for economic overheating, the intervening method of intervention is to decrease the energy. If the intervening has been done on $X$ by the increment $\Delta \varphi(X)=-\Delta<0$, the worst victim is the bane $K_{X}$ of $X$ which has the increment $\left(\rho_{1} \Delta\right)$. Thus the intervening principle of self-protection is to restore the bane $K_{X}$ of $X$ and the restoring method of selfprotection is to decrease the energy $\varphi\left(K_{X}\right)$ of the bane $K_{X}$ of $X$ by using the same intervention force on $X$ according to the intervention rule. In general, the decrease degree is $\left(-\rho_{3} \Delta\right)$ where $\rho_{3} \leq \rho_{1}$.

In mathematics, the following self-protection laws hold.

1) If $\Delta \varphi(X)=\Delta>0$, then the energy of subsystem $X_{K}$ will decrease the increment $\left(-\rho_{1} \Delta\right)$, which is the worst victim. So the capability of self-protection increases the energy of subsystem $X_{K}$ by increment $\left(\rho_{3} \Delta\right)$ where $\rho_{3} \leq \rho_{1}$, in order to restore the worst victim by according to the intervention rule.

2) If $\Delta \varphi(X)=-\Delta<0$, then the energy of subsystem $K_{X}$ will increase the increment $\left(\rho_{1} \Delta\right)$, which is the worst victim. So the capability of self-protection decreases the energy of subsystem $K_{X}$ by increment $\left(-\rho_{3} \Delta\right)$ where $\rho_{3} \leq \rho_{1}$, in order to restore the worst victim by according to the intervention rule.

In general, $0 \leq \rho_{3} \leq \rho_{1} \leq 1$. The $\rho_{1}$ is the intervention reaction coefficient. The $\rho_{3}$ is called a self-protection coefficient, which is used to represent the capability of self-protection. The larger $\rho_{3}$, the better the capability of self-protection. The state $\rho_{3}=\rho_{1}=1$ is the best state but the state $\rho_{3}=0$ is the worst state of selfprotection. According to the general economy of the protection principle, $\rho_{3}$ should be not greater than $\rho_{1}$ since the purpose of protection is to restore the victims and not reward the victims.

The self-protection rule can be explained as: the general principle of self-protection subsystem is that the most affected is protected firstly, the protection method and intervention force are in the same way.

In general, $\rho_{3}$ is also a decreasing function of the intervention force $\Delta$ since the worst victim is easily to restore all if $\Delta$ is small but the worst victim is not easily to restore all if $\Delta$ is large. The energy function of complex system, the stronger the more you use. In order to magnify $\rho_{3}$, should set up an economic society of the self-protection system, and often use it.

Theorem 3.1. Suppose that a steady multilateral system $V$ which has energy function $\varphi(*)$ and capabilities of intervention reaction and self-protection is with intervention reaction coefficients $\rho_{1}=\rho_{1}(\Delta)$ and $\rho_{2}=\rho_{2}(\Delta)$, and with self-protection coefficient $\rho_{3}=\rho_{3}(\Delta)$. If the capability of self-protection wants to restore both subsystems $X_{K}$ and $K_{X}$, then the following statements are true.

1) In the case of virtual disease, the treatment method is to increase the energy. If an intervention force on the subsystem $X$ of steady multilateral system $V$ is im- 
plemented such that its energy $\varphi(X)$ has been changed by increment $\Delta \varphi(X)=\Delta>0$, then all five subsystems will be changed finally by the increments as follows:

$$
\begin{aligned}
& \Delta \varphi(X)_{2}=\Delta \varphi(X)+\Delta \varphi(X)_{1}=\left(1-\rho_{2} \rho_{3}\right) \Delta>0, \\
& \Delta \varphi\left(X_{S}\right)_{2}=\Delta \varphi\left(X_{S}\right)+\Delta \varphi\left(X_{S}\right)_{1}=\left(\rho_{1}+\rho_{2} \rho_{3}\right) \Delta>0, \\
& \Delta \varphi\left(X_{K}\right)_{2}=\Delta \varphi\left(X_{K}\right)+\Delta \varphi\left(X_{K}\right)_{1}=-\left(\rho_{1}-\rho_{3}\right) \Delta \leq 0, \\
& \Delta \varphi\left(K_{X}\right)_{2}=\Delta \varphi\left(K_{X}\right)+\Delta \varphi\left(K_{X}\right)_{1}=-\left(\rho_{2}-\rho_{1} \rho_{3}\right) \Delta \leq 0, \\
& \Delta \varphi\left(S_{X}\right)_{2}=\Delta \varphi\left(S_{X}\right)+\Delta \varphi\left(S_{X}\right)_{1}=\left(\rho_{2}-\rho_{1} \rho_{3}\right) \Delta \geq 0 \\
& \forall \Delta \varphi(X)=\Delta>0 .
\end{aligned}
$$

2) In the case of real disease, the treatment method is to decrease the energy. If an intervention force on the subsystem $X$ of steady multilateral system $V$ is implemented such that its energy $\varphi(X)$ has been changed by increment $\Delta \varphi(X)=-\Delta<0$, then all five subsystems will be changed by the increments as follows:

$$
\begin{aligned}
& \Delta \varphi(X)_{2}=\Delta \varphi(X)+\Delta \varphi(X)_{1}=-\left(1-\rho_{2} \rho_{3}\right) \Delta<0, \\
& \Delta \varphi\left(X_{S}\right)_{2}=\Delta \varphi\left(X_{S}\right)+\Delta \varphi\left(X_{S}\right)_{1}=-\left(\rho_{2}-\rho_{1} \rho_{3}\right) \Delta \leq 0, \\
& \Delta \varphi\left(X_{K}\right)_{2}=\Delta \varphi\left(X_{K}\right)+\Delta \varphi\left(X_{K}\right)_{1}=\left(\rho_{2}-\rho_{1} \rho_{3}\right) \Delta \geq 0, \\
& \Delta \varphi\left(K_{X}\right)_{2}=\Delta \varphi\left(K_{X}\right)+\Delta \varphi\left(K_{X}\right)_{1}=\left(\rho_{1}-\rho_{3}\right) \Delta \geq 0, \\
& \Delta \varphi\left(S_{X}\right)_{2}=\Delta \varphi\left(S_{X}\right)+\Delta \varphi\left(S_{X}\right)_{1}=-\left(\rho_{1}+\rho_{2} \rho_{1}\right) \Delta<0 \\
& \forall \Delta \varphi(X)=-\Delta<0 .
\end{aligned}
$$

where the $\Delta \varphi\left({ }^{*}\right)_{1}$ 's are the increments under the capability of self-protection.\#

Corollary 3.1. Suppose that a steady multilateral system $V$ which has energy function $\varphi\left({ }^{*}\right)$ and capabilities of intervention reaction and self-protection is with intervention reaction coefficients $\rho_{1}=\rho_{1}(\Delta)$ and $\rho_{2}=\rho_{2}(\Delta)$, and with self-protection coefficient $\rho_{3}=\rho_{3}(\Delta)$. Then the capability of self-protection can make both subsystems $X_{K}$ and $K_{X}$ to be restored at the same time, i.e., the capability of self-protection is better, if and only if $\rho_{2}=\rho_{1} \rho_{3}$ and $\rho_{3}=\rho_{1}$.\#

Side effects of economic intervening problems were the question: in the economic intervening process, destroyed the normal balance of non-fall ill subsystem or non-intervention subsystem. By Theorem 3.1 and Corollary 3.1 , it can be seen that if the capability of self-protection of the steady multilateral system is better, i.e., the multilateral system has capability to protect all the victims to restore, then a necessary and sufficient condition is $\rho_{2}=\rho_{1} \rho_{3}$ and $\rho_{3}=\rho_{1}$. General for a stable complex system of economic society, the condition $\rho_{2}=\rho_{1} \rho_{3}$ is easy to meet since it can restore two subsystems by Theorem 3.1, but the condition $\rho_{3}=\rho_{1}$ is difficult to meet since it only can restore one subsystem by Theorem 3.1. At this point, the paper advocates the principle to avoid any side effect of intervening.

\subsection{Mathematical Reasoning of Intervening Principle by Using the Neighboring Relations of Steady Multilateral Systems}

Intervening principle by using the neighboring relations of steady multilateral systems is "Virtual disease for economic downturn is to fill his mother but real disease for economic overheating is to rush down his son". In order to show the rationality of the intervening principle, it is needed to prove the following theorems.

Theorem 3.2. Suppose that a steady multilateral system $V$ which has energy function and capabilities of intervention reaction and self-protection is with intervention reaction coefficients $\rho_{1}=\rho_{1}(\Delta)$ and $\rho_{2}=\rho_{2}(\Delta)$, and with self-protection coefficient $\rho_{3}=\rho_{3}(\Delta)$ satisfying $\rho_{2}=\rho_{1} \rho_{3}$ and $\rho_{3}=\rho_{1}$. Then the following statements are true.

In the case of virtual disease, if an intervention force on the subsystem $X$ of steady multilateral system $V$ is implemented such that its energy $\varphi(X)$ increases the increment $\Delta \varphi(X)=\Delta>0$, then the subsystems $S_{X}$, $X_{K}$ and $K_{X}$ can be restored at the same time, but the subsystems $X$ and $X_{S}$ will increase their energies by the increments

$$
\begin{aligned}
\Delta \varphi(X)_{2} & =\left(1-\rho_{2} \rho_{3}\right) \Delta \varphi(X) \\
& =\left(1-\rho_{2} \rho_{3}\right) \Delta=\left(1-\rho_{1}^{3}\right) \Delta>0
\end{aligned}
$$

and

$$
\begin{aligned}
\Delta \varphi\left(X_{S}\right)_{2} & =\left(\rho_{1}+\rho_{2} \rho_{3}\right) \Delta \varphi(X) \\
& =\left(\rho_{1}+\rho_{2} \rho_{3}\right) \Delta=\left(\rho_{1}+\rho_{1}^{3}\right) \Delta>0
\end{aligned}
$$

respectively.

On the other hand, in the case of real disease, if an intervention force on the subsystem $X$ of steady multilateral system $V$ is implemented such that its energy $\varphi(X)$ decreases, i.e., by the increment $\Delta \varphi(X)=-\Delta<0$, the subsystems $X_{S}, X_{K}$ and $K_{X}$ can also be restored at the same time, and the subsystems $X$ and $S_{X}$ will decrease their energies, i.e., by the increments

$$
\begin{aligned}
\Delta \varphi(X)_{2} & =\left(1-\rho_{2} \rho_{3}\right) \Delta \varphi(X) \\
& =-\left(1-\rho_{2} \rho_{3}\right) \Delta=-\left(1-\rho_{1}^{3}\right) \Delta<0
\end{aligned}
$$

and

$$
\begin{aligned}
\Delta \varphi\left(S_{X}\right)_{2} & =\left(\rho_{1}+\rho_{2} \rho_{3}\right) \Delta \varphi(X) \\
& =-\left(\rho_{1}+\rho_{2} \rho_{3}\right) \Delta=-\left(\rho_{1}+\rho_{1}^{3}\right) \Delta<0
\end{aligned}
$$

respectively.\#

Theorem 3.3. For a steady multilateral system $V$ which has energy function $\varphi(*)$ and capabilities of intervening reaction and self-protection, assume inter- 
vention reaction coefficients are $\rho_{1}$ and $\rho_{2}$, and let the self-protection coefficient be $\rho_{3}$, which satisfy $\rho_{2}=\rho_{1} \rho_{3}, \rho_{3}=\rho_{1}$ and $\rho_{1} \geq \rho_{0}$ where

$\rho_{0} \approx(<) 0.5897545123$ (the following the same) is the solution of $2 \rho_{1}^{3}+\rho_{1}=1$. Then the following statements are true.

1) In the case of virtual disease, if an intervention force on the subsystem $X$ of steady multilateral system $V$ is implemented such that its energy $\varphi(X)$ has been changed by increment $\Delta \varphi(X)=\Delta>0$, then the final increment $\left(\rho_{1}+\rho_{2} \rho_{3}\right) \Delta$ of the energy $\varphi\left(X_{S}\right)$ of the subsystem $X_{S}$ changed is greater than or equal to the final increment $\left(1-\rho_{2} \rho_{3}\right) \Delta$ of the energy $\varphi(X)$ of the subsystem $X$ changed based on the capability of self-protection.

2) In the case of real disease, if an intervention force on the subsystem $X$ of steady multilateral system $V$ is implemented such that its energy $\varphi(X)$ has been changed by increment $\Delta \varphi(X)=-\Delta<0$, then the final increment $-\left(\rho_{1}+\rho_{2} \rho_{3}\right) \Delta$ of the energy $\varphi\left(S_{X}\right)$ of the subsystem $S_{X}$ changed is less than or equal to the final increment $-\left(1-\rho_{2} \rho_{3}\right) \Delta$ of the energy $\varphi(X)$ of the subsystem $X$ changed based on the capability of selfprotection. \#

Corollary 3.2. For a steady multilateral system $V$ which has energy function $\varphi(*)$ and capabilities of intervening reaction and self-protection, assume intervention reaction coefficients are $\rho_{1}$ and $\rho_{2}$, and let the self-protection coefficient be $\rho_{3}$, which satisfy

$\rho_{2}=\rho_{1} \rho_{3}, \rho_{3}=\rho_{1}$ and $\rho_{1}<\rho_{0}$. Then the following statements are true.

1) In the case of virtual disease, if an intervention force on the subsystem $X$ of steady multilateral system $V$ is implemented such that its energy $\varphi(X)$ has been changed by increment $\Delta \varphi(X)=\Delta>0$, then the final increment $\left(\rho_{1}+\rho_{2} \rho_{3}\right) \Delta$ of the energy $\varphi\left(X_{S}\right)$ of the subsystem $X_{S}$ changed is less than the final increment $\left(1-\rho_{2} \rho_{3}\right) \Delta$ of the energy $\varphi(X)$ of the subsystem $X$ changed based on the capability of self-protection.

2) In the case of real disease, if an intervention force on the subsystem $X$ of steady multilateral system $\mathrm{V}$ is implemented such that such that its energy $\varphi(X)$ has been changed by increment $\Delta \varphi(X)=-\Delta<0$, then the final increment $-\left(\rho_{1}+\rho_{2} \rho_{3}\right) \Delta$ of the energy $\varphi\left(S_{X}\right)$ of the subsystem $S_{X}$ changed is greater than the final increment $-\left(1-\rho_{2} \rho_{3}\right) \Delta$ of the energy $\varphi(X)$ of the subsystem $X$ changed based on the capability of selfprotection.\#

By Theorems 3.2 and 3.3 and Corollary 3.2, the intervention method of "Virtual disease for economic downturn is to fill his mother but real disease for economic overheating is to rush down his son" should be often used in case: $\rho_{2}=\rho_{1} \rho_{3}, \rho_{3}=\rho_{1}$ and $\rho_{1} \geq \rho_{0}$ since in this time, $\left(\rho_{1}+\rho_{2} \rho_{3}\right) \Delta \geq\left(1-\rho_{2} \rho_{3}\right) \Delta$.

\subsection{Mathematical Reasoning of Intervening Principle by Using the Alternate Relations of Steady Multilateral Systems}

Intervening principle by using the alternate relations of steady multilateral systems is "Strong inhibition of the same time, support the weak". In order to show the rationality of the intervening Principle, it is needed to prove the following theorems.

Theorem 3.4. Suppose that a steady multilateral system $V$ which has energy function $\varphi(*)$ and capabilities of intervention reaction and self-protection is with intervention reaction coefficients $\rho_{1}=\rho_{1}(\Delta)$ and $\rho_{2}=\rho_{2}(\Delta)$, and with self-protection coefficient $\rho_{3}=\rho_{3}(\Delta)$. Then the following statements are true.

Assume there are two subsystems $X$ and $X_{K}$ of $V$ with an alternate relation such that $X$ encounters virtual disease, and at the same time, $X_{K}$ befalls real disease. If an intervention force on the subsystem $X$ of steady multilateral system $V$ is implemented such that its energy $\varphi(X)$ has been changed by increment $\Delta \varphi(X)=\Delta>0$, and at the same time, another intervention force on the subsystem $X_{K}$ of steady multilateral system $V$ is also implemented such that its energy $\varphi\left(X_{K}\right)$ has been changed by increment $\Delta \varphi\left(X_{K}\right)=-\Delta<0$, then all other subsystems: $S_{X}, K_{X}$ and $X_{S}$ can be restored at the same time, and the subsystems $X$ and $X_{K}$ will increase and decrease their energies by the same size but the direction opposite, i.e., by the increments

$$
\begin{aligned}
\Delta \varphi(X)_{3} & =\left(1-\rho_{2} \rho_{3}\right) \Delta \varphi(X) \\
& =\left(1-\rho_{2} \rho_{3}\right) \Delta=\left(1-\rho_{1}^{3}\right) \Delta>0
\end{aligned}
$$

and

$$
\begin{aligned}
\Delta \varphi\left(X_{K}\right)_{3} & =\left(1-\rho_{2} \rho_{3}\right) \Delta \varphi\left(X_{K}\right) \\
& =-\left(1-\rho_{2} \rho_{3}\right) \Delta=-\left(1-\rho_{1}^{3}\right) \Delta<0
\end{aligned}
$$

respectively.

Assume there are two subsystems $X$ and $K_{X}$ of $V$ with an alternate relation such that $X$ encounters real disease, and at the same time, $K_{X}$ befalls virtual disease. If an intervention force on the subsystem $X$ of steady multilateral system $V$ is implemented such that its energy $\varphi(X)$ has been changed by increment $\Delta \varphi(X)=-\Delta<0$, and at the same time, another intervention force on the subsystem $K_{X}$ of steady multilateral system $V$ is also implemented such that its energy $\varphi\left(K_{X}\right)$ has been changed by increment $\varphi\left(K_{X}\right)=\Delta>0$, then all other subsystems: $S_{X}, X_{K}$ and $X_{S}$ can be restored at the same time, and the subsystems $X$ and $K_{X}$ will decrease and increase their energies by the same size but the direction opposite, i.e., by the increments 


$$
\begin{aligned}
\Delta \varphi(X)_{3} & =\left(1-\rho_{2} \rho_{3}\right) \Delta \varphi(X) \\
& =-\left(1-\rho_{2} \rho_{3}\right) \Delta=-\left(1-\rho_{1}^{3}\right) \Delta<0
\end{aligned}
$$

and

$$
\begin{aligned}
\Delta \varphi\left(K_{X}\right)_{3} & =\left(1-\rho_{2} \rho_{3}\right) \Delta \varphi\left(K_{X}\right) \\
& =\left(1-\rho_{2} \rho_{3}\right) \Delta=\left(1-\rho_{1}^{3}\right) \Delta>0
\end{aligned}
$$

respectively. \#

By Theorems 3.3 and 3.4 and Corollary 3.2, the method of "Strong inhibition of the same time, support the weak" should be used in case: $\rho_{2}=\rho_{1} \rho_{3}, \rho_{3}=\rho_{1}$ and $\rho_{1}<\rho_{0}$ since $\left(\rho_{1}+\rho_{2} \rho_{3}\right) \Delta<\left(1-\rho_{2} \rho_{3}\right) \Delta$.

\section{Rationality of Intervening Principle of Traditional Chinese Economics and "Yin Yang Wu Xing” Theory}

\subsection{Traditional Chinese Economics and "Yin Yang Wu Xing” Theory}

Ancient Chinese "Yin Yang Wu Xing" [38] Theory has been surviving for several thousands of years without dying out, proving it reasonable to some extent. If we regard as the same category, the neighboring relation $\rightarrow$ as beneficial, harmony, obedient, loving, etc. and the alternate relation $\Rightarrow$ as harmful, conflict, ruinous, killing, etc., then the above defined stable logic analysis model is similar to the logic architecture of reasoning of "Yin Yang Wu Xing". Both "Yin" and "Yang" mean that there are two opposite relations in the world: harmony or loving $\rightarrow$ and conflict or killing $\Rightarrow$, as well as a general equivalent category $\sim$. There is only one of three relations $\sim, \rightarrow$ and $\Rightarrow$ between every two objects. Everything $X \neq \varnothing$ makes something $X_{S} \neq \varnothing$, and is made by something $S_{X} \neq \varnothing$; Everything restrains something $X_{K} \neq \varnothing$, and is restrained by something $K_{X} \neq \varnothing$; i.e., one thing overcomes another thing and one thing is overcome by another thing. The ever changing world $V$, following the relations: $\sim, \rightarrow$ and $\Rightarrow$, must be divided into five categories by the equivalent relation , being called "Wu Xing": $\operatorname{wood}(X)$, fire $\left(X_{S}\right)$, soil $\left(X_{K}\right)$, metal $\left(K_{X}\right)$, water $\left(S_{X}\right)$. The "Wu Xing" is to be "neighbor is friend": $\operatorname{wood}(X) \rightarrow$ fire $\left(X_{S}\right) \rightarrow$ soil $\left(X_{K}\right) \rightarrow$ metal $\left(K_{X}\right) \rightarrow$ water $\left(S_{X}\right) \rightarrow$ wood $(X)$, and "alternate is foe": $\operatorname{wood}(X) \Rightarrow \operatorname{soil}\left(X_{K}\right)$ $\Rightarrow$ water $\left(S_{X}\right) \Rightarrow$ fire $\left(X_{S}\right) \Rightarrow \operatorname{metal}\left(K_{X}\right) \Rightarrow$ $\operatorname{wood}(X)$. In other words, the ever changing world must be divided into five categories:

$$
V=X+X_{S}+X_{K}+K_{X}+S_{X}
$$

Satisfying

$$
X \rightarrow X_{S} \rightarrow X_{K} \rightarrow K_{X} \rightarrow S_{X} \rightarrow X
$$

And

$$
X \Rightarrow X_{K} \Rightarrow S_{X} \Rightarrow X_{S} \Rightarrow K_{X} \Rightarrow X
$$

where elements in the same category are equivalent to one another. We can see, from this, the ancient Chinese "Yin Yang Wu Xing" theory is a reasonable logic analysis model to identify the stability and relationship of complex economic systems.

TCE firstly use the verifying relationship method of "Yin Yang Wu Xing" Theory to explain the relationship between economic society and environment. Secondly, based on "Yin Yang Wu Xing" Theory, the relations of development processes of economic society can be shown by the neighboring relation and alternate relation of five subsets. Then a normal economic society can be shown as a steady multilateral system since there are the loving relation and the killing relation and the liking relation. The loving relation in TCE can be explained as the neighboring relation, called "Sheng (生)". The killing relation in TCE can be explained as the alternate relation, called "Ke (克)". The liking relation can be explained as the equivalent relation, called "Tong-Lei (同类)". Constraints and conversion between five subsets are equivalent to the two kinds of triangle reasoning. So a normal economic society can be classified into five equivalence classes.

For example, in TCE, an economic complex system is similar to a human body. A normal economic society following the "Yin Yang Wu Xing" Theory was classified into five equivalence classes as follows:

wood $(X)=$ industry, legislature, cognitive, structure, goods, spring, birth $\}$;

fire $\left(X_{S}\right)=$ agriculture, administrative institutions, development, fluctuations, foods, summer, growth $\}$;

soil $\left(X_{K}\right)=\{$ commerce, media organizations, coordination, harmonious, money, long-summer, combined $\}$;

metal $\left(K_{X}\right)=$ \{education, non-governmental institutions, function, quality, knowledge, autumn, accept $\}$;

water $\left(S_{X}\right)=$ army, supervisory organ, risk, cost, weapons, winter, hiding $\}$.

There is only one of both loving and hating (or killing) relations between every two classes. Generally speaking, close is love, alternate is hate.

In every category of internal, think that they are equivalent relationship, between each two of their elements there is a force of similar material accumulation of each other. It is because their pursuit of the goal is the same, i.e., follows the same "Axiom system". It can increase the energy of the class if they accumulate together. Any nature material activity follows the principle of maximizing so energy. In general, the size of the force of similar material accumulation of each other is smaller than the size of the loving force or the killing force in a stable economic complex system. The stability of any economic complex system first needs to maintain the equilibrium of the killing force and the loving force. For 
a stable economic complex system, if the killing force is large, i.e., $\rho_{1}$ becomes larger, then the loving force is large and the force of similar material accumulation of each other is also large. They can make the economic complex system more stable. If the killing force is small, i.e., $\rho_{1}$ becomes smaller, then the loving force is small and the force of similar material accumulation of each other is also small. They can make the economic complex system becoming unstable.

It has been shown in Theorems 2.1-2.4 that the classification of five subsets is quite possible based on the mathematical logic. As for the characteristics of the five subsets is reasonable or not. It is need more research work. It has been also shown in Theorems 3.1-3.4 that the logical basis of TCE is a steady multilateral system.

The vigor energy (or force, essence, Chi, spirit) of TCE means the energy function in a steady multilateral system.

There are two kinds of economic disease in TCE: Real disease or economic overheating and virtual disease or economic downturn. They generally mean the subsystem is abnormal, its energy is too high as real disease for economic overheating; or its energy is too low as virtual disease for economic downturn.

The intervening method of TCE is to "xie Chi" which means to rush down the energy if a real disease for economic overheating is treated, or to "bu Chi" which means to fill the energy if a virtual disease for economic downturn is treated. Like intervening the subsystem, decrease when the energy is too high, increase when the energy is too low.

Both the capability of intervention reaction and the capability of self-protection of the multilateral system are equivalent to the Immunization of TCE. This capability is really existence for a complex economic society. Its target is to protect other economic subsystem while treating one economic subsystem. It is because if the capability is not existence, then $\rho_{1}=\rho_{2}=\rho_{3}=0$. In this time, the energy of the system will be the sum of energy of each part. Thus the economic system will be a simple economic system which is not what we consider range.

\subsection{Intervening Principle If Only One Subsystem of the Economic Society Falls Ill}

If we always intervene the abnormal subsystem of the economic society system directly, the intervention method always destroy the balance of the economic society system because it is having strong side effects to the mother or the son of the subsystem which may be nondisease of economic subsystem or non-intervened subsystem by using Theorem 3.2. The intervening method also will decrease the capability of intervention reaction because the method which doesn't use the capability of intervention reaction makes the $\rho_{1}$ and $\rho_{2}$ near to 0 . The state $\rho_{1}=\rho_{2}=0$ is the worst state of the economic society system, namely Economic Crisis. On the way, the economic intervening resistance problem will occur since any economic intervening method is possible too little for some small $\rho_{1}$ and $\rho_{2}$.

But, by Corollary 3.2, it will even be better if we intervene subsystem $X$ itself directly when $\rho_{2}=\rho_{1} \rho_{3}$, $\rho_{3}=\rho_{1}$ and $\rho_{1}<\rho_{0}$. In this case:

$$
\rho_{1}+\rho_{2}<0.9375648971
$$

It can be explained that if a multilateral system which has a poor capability of intervention reaction, then it is better to intervene the subsystem itself directly than indirectly. But similar to above, the intervention method always destroys the balance of multilateral systems such that there is at least one side effect occurring. And the intervention method also has harmful to the capability of intervention reaction making the economic intervening resistance problem also occur. Therefore the intervention method directly can be used in case $\rho_{2}=\rho_{1} \rho_{3}, \rho_{3}=\rho_{1}$ and $\rho_{1}<\rho_{0}$ but should be used as little as possible.

If we always intervene the abnormal subsystem of the economic society system indirectly, the intervention method can be to maintain the balance of the economic society system because it has not any side effect to all other subsystems which are not both the economic disease subsystem and the intervened subsystem by using Theorem 3.1. The intervening method also increases the capability of intervention reaction because the method of using the intervention reaction makes the $\rho_{1}$ and $\rho_{2}$ near to 1 . The state $\rho_{1}=\rho_{2}=1$ is the best state of the economic society system. On the way, it is almost none economic intervening resistance problem since any economic intervening method is possible good for some large $\rho_{1}$ and $\rho_{2}$.

For example, in China, many local agricultural development is insufficient (i.e., $X_{S}$ falls virtual disease), the local frequently used method is to use industrial keep agriculture (i.e., to increase the energy of $X$ which is the mother of $X_{S}$ ). The idea is precisely "Virtual disease for economic downturn is to fill his mother" if one subsystem of economic society falls virtual ill.

All in all, the economic society system satisfies the intervention rule and the self-protection rule. It is said a healthy economic society if the intervention reaction coefficient $\rho_{1}$ satisfies $\rho_{1} \geq \rho_{0}$. In logic and practice, it's reasonable $\rho_{1}+\rho_{2}$ near to 1 since an economic output subsystem is absolutely necessary social other subsystems of all consumption. In case: $\rho_{1}+\rho_{2}=1$, all the energy for intervening economic society subsystem can transmit to other economic society subsystems which have neighboring relations or alternate relations with the 
intervening economic society subsystem. The condition $\rho_{1} \geq \rho_{0}$ can be satisfied when $\rho_{2}=\rho_{1} \rho_{3}$ and $\rho_{3}=\rho_{1}$ for an economic society since $\rho_{1}+\rho_{2}=1$ implies

$$
\rho_{1}=(\sqrt{5}-1) / 2 \approx 0.618>\rho_{0}
$$

and

$$
\rho_{2}=1-(\sqrt{5}-1) / 2 \approx 0.382
$$

If this assumptions is set up, then the intervening principle: "Real disease for economic downturn is to rush down his son and virtual disease for economic downturn is to fill his mother" based on "Yin Yang Wu Xing" Theory in TCE, is quite reasonable. But, in general, the ability of self-protection often is insufficient for an usual economic society, i.e., $\rho_{3}$ is small. A common standard is $\rho_{3}=\frac{1-\rho_{1}}{2 \rho_{2}} \approx \frac{1}{2}$, i.e., there is a principle which all losses are bear in economic society. Thus the general condition often is $\rho_{1} \approx 0.618 \geq \rho_{3} \approx 0.5 \geq \rho_{2} \approx 0.382$. Interesting, they near to the golden numbers.

On the other hand, in TCE, real disease for economic overheating and virtual disease for economic downturn has their reasons. Real disease for economic overheating is caused by the born subsystem and virtual disease for economic downturn is caused by the bear subsystem. Although the reason can not be proved easily in mathematics or experiments, the intervening method under the assumption is quite equal to the intervening method in the intervention indirectly. It has also proved that the economic intervening principle is true from the other side.

\subsection{Intervening Principle If Only Two Subsystems with the Loving Relation of the Economic Society System Encounter Sick}

Suppose that the two subsystems $X$ and $X_{S}$ of the economic society system are abnormal (economic downturn or overheating). In the economic society of two noncompatible relations with constraints, only two situations may occur:

1) $X$ encounters virtual disease for economic downturn, and at the same time, $X_{S}$ befalls virtual disease for economic downturn, i.e., the energy of $X$ is too low and the energy of $X_{S}$ is also too low. It is because $X$ bears $X_{S}$. The economic downturn causal is $X$.

2) $X$ encounters real disease for economic overheating, and at the same time, $X_{S}$ befalls real disease for economic overheating, i.e., the energy of $X$ is too high and the energy of $X_{S}$ is also too high. It is because $X_{S}$ is born by $X$. The economic downturn causal is $X_{S}$.

It can be shown by Theorem 3.3 that when intervention reaction and self-protection coefficients satisfy that $\rho_{2}=\rho_{1} \rho_{3}, \quad \rho_{3}=\rho_{1}$ and $\rho_{1} \geq \rho_{0}$, if one wants to treat the abnormal subsystems $X$ and $X_{S}$, then

1) In virtual disease for economic downturn, the one should intervene subsystem $X$ directly by increasing its energy. It means "virtual disease for economic downturn is to fill his mother" because the economic downturn causal is $X$;

2) In real disease for economic overheating, the one should intervene subsystem $X_{S}$ directly by decreasing its energy. It means "Real disease for economic overheating is to rush down his son" because the economic downturn causal is $X_{S}$.

For example, in China, many local the money $\left(X_{K}\right)$ is virtual (currency devaluation) and the food $\left(X_{S}\right)$ is also virtual (inflation of food prices), the local frequently used method is mainly to increase the supply of food (i.e., to increase the energy of $X_{S}$ which is the mother of $X_{K}$ ), in order to control the price of food cannot rise. The idea is precisely "Virtual disease for economic downturn is to fill his mother" if two loving subsystems $X_{S}$ (as $X$ ) and $X_{K} \quad\left(\right.$ as $\left.X_{S}\right)$ of economic society fall virtual ill.

The intervention method can be to maintain the balance of the economic society because only two economic downturn subsystems are treated, by using Theorem 3.2, such that there is not any side effect for all other subsystems. And the intervention method can also be to enhance the capability of intervention reaction because the method of using intervention reaction makes the $\rho_{1}$ and $\rho_{2}$ greater and near to 1 . The state $\rho_{1}=\rho_{2}=1$ is the best state of the economic society system. On the way, it almost have none economic intervening resistance problem since any economic intervening method is possible good for some large $\rho_{1}$ and $\rho_{2}$.

\subsection{Intervening Principle If Only Two Subsystems with the Killing Relation of the Economic Society System Encounter Sick}

Suppose that the subsystems $X$ and $X_{K}$ of an economic society system are abnormal (economic downturn or overheating). In the economic society system with the constraints of two non-compatible relations, only a situation may occur: $X$ encounters virtual disease for economic downturn, and at the same time, $X_{K}$ befalls real disease for economic overheating, i.e., the energy of $X$ is too low and the energy of $X_{K}$ is too high. The disease is serious because $X_{K}$ has harmed the $X$ by using the method of incest such that the king relation between $X$ and $X_{K}$ is damaged.

It can be shown by Theorem 3.4 that when intervention reaction and self-protection coefficients satisfy $\rho_{2}=\rho_{1} \rho_{3}, \quad \rho_{3}=\rho_{1}$ and $\rho_{1}<\rho_{0}$, if one wants to treat the abnormal subsystems $X$ and $X_{K}$, the one should intervene subsystem $X$ directly by increasing its en- 
ergy, and at the same time, intervene subsystem $X_{K}$ directly by decreasing its energy. It means that "Strong inhibition of the same time, support the weak".

For example, thirty years ago, China's social coordination function is very good, society is rife with average socialist, and industry is very poor. In addition, the economic society is not rich. In other words, $X$ falls virtual disease and at the same time, $X_{K}$ befalls real disease. The disease is serious because $X_{K}$ has harmed the $X$ by using the method of incest such that $X$ cannot kill $X_{K}$, which has damaged the king relation between $X$ and $X_{K}$ of the economic society. The condition that $\rho_{2}=\rho_{1} \rho_{3}, \rho_{3}=\rho_{1}$ and $\rho_{1}<\rho_{0}$ can think it is true since the economic society is not rich although social intervention response ability and self-protection ability is good. In order to cure the serious disease, Deng XiaoPing's taking method is to break the "iron bowl" (to fill up $X$, strengthen legislation and industrial income), and to allow a few people to get rich (to rush down $X_{K}$, abate the coordinated ability). The idea is "Strong inhibition of the same time, support the weak" if $X$ falls virtual disease and at the same time, $X_{K}$ befalls real disease.

The intervention method can be to maintain the balance of economic society system because only two economic downturn subsystems are treated, by using Theorem 3.4, such that there is not any side effect for all other subsystems. And the intervention method can also be to enhance the capability of self-protection because the method of using the capability of self-protection makes the $\rho_{3}$ and $\rho_{1}$ greater and near to 1 . The state $\rho_{3}=\rho_{1}=1$ is the best state of the steady multilateral system. On the way, it almost have none economic intervening resistance problem since any economic intervening method is possible good for some large $\rho_{3}$ and $\rho_{1}$.

\section{Conclusions}

This work shows how to treat the economic diseases (downturn or overheating) of an economic society system and three methods are presented.

If only one subsystem falls ill, mainly the intervening method should be to intervene it indirectly for case: $\rho_{2}=\rho_{1} \rho_{3}, \quad \rho_{3}=\rho_{1}$ and $\rho_{1} \geq \rho_{0}$, according to the intervening principle of "Real disease for economic overheating is to rush down his son but virtual disease for economic downturn is to fill his mother". The intervention method directly can be used in case:

$\rho_{2}=\rho_{1} \rho_{3}, \rho_{3}=\rho_{1}$ and $\rho_{1}<\rho_{0}$ but should be used as little as possible.

If two subsystems with the loving relation encounter sick, the intervening method should be intervene them directly also according to the intervening principle of "Real disease for economic overheating is to rush down his son but virtual disease for economic downturn is to fill his mother".
If two subsystems with the killing relation encounter sick, the intervening method should be intervene them directly according to the intervening principle of "Strong inhibition of the same time, support the weak".

Other properties, such as balanced, orderly nature of Wu-Xing, and so on, will be discussed in the next articles.

\section{Acknowledgements}

This article has been repeatedly invited as reports, such as The Renmin University of China, Beijing Normal University, Fudan University, Shanxi University, Xuchang College, and so on. The work was supported by Specialized Research Fund for the Doctoral Program of Higher Education of Ministry of Education of China (Grant No. 200802691021).

\section{REFERENCES}

[1] Y. S. Zhang, "Theory of Multilateral Matrices," Chinese State Press, 1993. http://www.mlmatrix.com

[2] Y. S. Zhang, "Theory of Multilateral Systems," 2007. http://www.mlmatrix.com

[3] Y. S. Zhang, "Mathematical Reasoning of Treatment Principle Based on 'Yin Yang Wu Xing' Theory in Traditional Chinese Medicine," Chinese Medicine, Vol. 2, No. 1, 2011, pp. 6-15. doi:10.4236/cm.2011.21002

[4] Y. S. Zhang, "Mathematical Reasoning of Treatment Principle Based on 'Yin Yang Wu Xing' Theory in Traditional Chinese Medicine(II)," Chinese Medicine, Vol. 2, No. 4, 2012, pp. 158-170.

[5] Y. S. Zhang, "Mathematical Reasoning of Treatment Principle Based on the Stable Logic Analysis Model of Complex Systems," Intelligent Control and Automation, Vol. 3, No. 1, 2012, pp. 6-15.

[6] Y. S. Zhang and W. L. Shao, "Image MathematicsMathematical Intervening Principle Based on 'Yin Yang Wu Xing' Theory in Traditional Chinese Mathematics (I)," Applied Mathematics, Vol. 3 No. 6, 2012, pp. 617636. doi:10.4236/am.2012.36096

[7] Y. S. Zhang, S. S. Mao, C. Z. Zhan and Z. G. Zheng, "Stable Structure of the Logic Model with Two Causal Effects," Chinese Journal of Applied Probability and Statistics, Vol. 21, No. 4, 2005, pp. 366-374.

[8] C. Luo, X. P. Chen and Y. S. Zhang, "The Turning Point Analysis of Finance Time Series," Chinese Journal of Applied Probability and Statistics, Vol. 26, No. 4, 2010, pp. 437-442.

[9] Y. S. Zhang, X. Q. Zhang and S. Y. Li, "SAS Language Guide and Application," Shanxi People's Press, Shanxi, 2011.

[10] Y. S. Zhang and S. S. Mao, "The Origin and Development Philosophy Theory of Statistics," Statistical Research, Vol. 12, 2004, pp. 52-59.

[11] C. Luo, X. L. Zhang and Y. S. Zhang, "Symmetrical Frame Isomorphism Class Count-The New Thinking of Dealing with Complex Systems Series Eight," Journal of 
Shanghai Institute of Technology (Natural Science) Vol. 11, No. 3, 2011, pp. 253-261.

[12] N. Q. Feng, Y. H. Qiu, F. Wang, Y. S. Zhang and S. Q. Yin, "A Logic Analysis Model about Complex System's Stability: Enlightenment From Nature," Lecture Notes in Computer Science, Vol. 3644, 2005, pp. 828-838. doi:10.1007/11538059 86

[13] N. Q. Feng, Y. H. Qiu, Y. S. Zhang, F. Wang and Y. He, "A Intelligent Inference Model about Complex System's Stability: Inspiration from Nature," International Joural of Intelligent Technique, Vol. 1, 2005, pp. 1-6.

[14] N. Q. Feng, Y. H. Qiu, Y. S. Zhang, C. Z. Zhan and Z. G. Zheng, "A Logic Analysis Model of Stability of Complex System Based on Ecology," Computer Science, Vol. 33, No. 7, 2006, pp. 213-216.

[15] C. Y. Pan, X. P. Chen, Y. S. Zhang and S. S. Mao, "Logical Model of Five-Element Theory in Chinese Traditional Medicine," Journal of Chinese Modern Traditional Chinese Medicine, Vol. 4, No. 3, 2008, pp. 193-196.

[16] X. P. Chen, W. J. Zhu, C. Y. Pan and Y. S. Zhang, "Multilateral System," Journal of Computer and System Sciences, Vol. 17, No. 1, 2009, pp. 55-57.

[17] C. Luo and Y. S. Zhang, "Framework Definition and Partition Theorems Dealing with Complex Systems: One of the Series of New Thinking," Journal of Shanghai Institute of Technology (Natural Science), Vol. 10, No. 2, 2010, pp. 109-114.

[18] C. Luo and Y. S. Zhang, "Framework and Orthogonal Arrays: the New Thinking of Dealing with Complex Systems Series Two," Journal of Shanghai Institute of Technology (Natural Science), Vol. 10, No. 3, 2010, pp. 159-163.

[19] J. Y. Liao, J. J. Zhang and Y. S. Zhang, "Robust Parameter Design on Launching an Object to Goal," Mathematics in Practice and Theory, Vol. 40, No. 24, 2010, pp 126-132.

[20] Y. S. Zhang, S. Q. Pang, Z. M. Jiao and W. Z. Zhao, "Group Partition and Systems of Orthogonal Idempotents," Linear Algebra and Its Applications, Vol. 278, No. 1-3, 1998, pp. 249-262.

[21] J. L. Zhao and Y. S. Zhang, "The Characteristic Description of Idempotent Orthogonal Class System," Advances in Matrix Theory and Its Applications, Proceedings of the Eighth International Conference on Matrix and Its Applications, World Academic Press, Taiyuan, Vol. 1, No. 1, 2008, pp. 445-448.

[22] X. P. Chen, C. Y. Pan and Y. S. Zhang, "Partitioning the Multivariate Function Space into Symmetrical Classes," Mathematics in Practice and Theory, Vol. 39, No. 2, 2009, pp. 167-173.

[23] C. Y. Pan, X. P. Chen and Y. S. Zhang, "Construct Systems of Orthogonal Idempotents," Journal of East China University (Natural Science), Vol. 141, No. 5, 2008, pp. 51-58.

[24] C. Y. Pan, H. N. Ma, X. P. Chen and Y. S. Zhang, "Proof Procedure of Some Theories in Statistical Analysis of Global Symmetry," Journal of East China Normal University (Natural Science), Vol. 142, No. 5, 2009, pp. 127137.
[25] X. Q. Zhang, Y. S. Zhang and S. S. Mao, "Statistical Analysis of 2-Level Orthogonal Satursted Designs: The Procedure of Searching Zero Effects," Journal of East China Normal University (Natural Science), Vol. 24, No. 1, 2007, pp. 51-59.

[26] Y. S. Zhang, Y. Q. Lu and S. Q. Pang, "Orthogonal Arrays Obtained by Orthogonal Decomposition of Projection Matrices," Statistica Sinica, Vol. 9, 1999, pp. 595604.

[27] Y. S. Zhang, S. Q. Pang and Y. P. Wang, "Orthogonal Arrays Obtained by Generalized Hadamard Product," Discrete Math, Vol. 238, No. 1-3, 2001, pp. 151-170. doi:10.1016/S0012-365X(00)00421-0

[28] Y. S. Zhang, L. Duan, Y. Q. Lu and Z. G. Zheng, "Construction of Generalized Hadamard Matrices $D\left(r^{m}(r+1), r^{m}(r+1) ; p\right)$," Journal of Statistical Planning and Inference, Vol. 104, No. 2, 2002, pp. 239-258. doi:10.1016/S0378-3758(01)00249-X

[29] Y. S. Zhang, "Data Analysis and Construction of Orthogonal Arrays," East China Normal University, 2006.

[30] Y. S. Zhang, "Orthogonal Arrays Obtained by RepeatingColumn Difference Matrices," Discrete Mathematics, Vol. 307, No. 2, 2007, pp. 246-261. doi:10.1016/j.disc.2006.06.029

[31] X. D. Wang, Y. C. Tang, X. P. Chen and Y. S. Zhang, "Design of Experiment in Global Sensitivity Analysis Based on ANOVA High-Dimensional Model Representation," Communication in Statistics: Simulation and Computation, Vol. 39, No. 6, 2010, pp. 1183-1195. doi:10.1080/03610918.2010.484122

[32] X. D. Wang, Y. C. Tang and Y. S. Zhang, "Orthogonal Arrays for the Estimation of Global Sensitivity Indices Based on ANOVA High-Dimensional Model Representation," Communication in Statistics: Simulation and Computation, Vol. 40, No. 9, 2011, pp. 1324-1341. doi:10.1080/03610918.2011.575500

[33] J. T. Tian, Y. S. Zhang, Z. Q. Zhang, C. Y. Pan and Y. Y. Gan, "The Comparison and Application of Balanced Block Orthogonal Arrays and Orthogonal Arrays," Journal of Mathematics in Practice and Theory, Vol. 39, No. 22, 2009, pp. 59-67.

[34] C. Luo and C. Y. Pan, "Method of Exhaustion to Search Orthogonal Balanced Block Designs," Chinese Journal of Applied Probability and Statistics, Vol. 27, No. 1, 2011, pp. 1-13.

[35] Y. S. Zhang, W. G. Li, S. S. Mao and Z. G. Zheng, “Orthogonal Arrays Obtained by Generalized Difference Matrices with G Levels," Science China Mathematics, Vol. 54, No. 1, 2011, pp. 133-143. doi:10.1007/s11425-010-4144-y

[36] Lao-tzu, "Tao Te Ching,” 2010. http://acc6.its.brooklyn.cuny.edu.

[37] M.-J. Cheng, "Lao-Tzu, My Words Are Very Easy to Understand: Lectures on the Tao Teh Ching," North Atlantic Books, Richmond, 1981.

[38] Research Center for Chinese and Foreign Celebrities and Developing Center of Chinese Culture Resources, "Chinese Philosophy Encyclopedia," Shanghai People Press, Shanghai, 1994. 